\section{A abordagem pedagógica em Teoria Sentido-Texto: descrição de colocação lexical especializada da Hemodinâmica}

The pedagogical approach in Meaning-Text Theory: description of specialized lexical collocation in the

Hemodynamics

Caroline de Castro PIRES (UFRGS) caroline.castro@alvorada.ifrs.edu.br

Recebido em: 31 de ago. de 2020. Aceito em: 21 de set. de 2020.
PIRES, Caroline de Castro. A

abordagem pedagógica em Teoria

Sentido-Texto: descrição de

colocação lexical especializada da

Hemodinâmica. Entrepalavras,

Fortaleza, v. 11, n. esp., p. 180-195,

ago. 2021. DOI: $10.22168 / 2237-6321-$

$10 \operatorname{esp} 2062$.

Resumo: O presente artigo insere-se nos estudos sobre o uso de abordagens pedagógicas ao que se refere às ferramentas lexicográficas de descrição de vocabulário especializado, se considerado o processo de aprendizagem lexical. No entanto, diferentemente de propor produtos lexicográficos que visam à aprendizagem de uma segunda língua, especificamente, com vistas à proficiência linguística nos moldes do ensino de língua estrangeira, neste artigo, se optou por tentar utilizar mecanismos já descritos na literatura especializada para apresentar uma abordagem pedagógica no âmbito da Teoria SentidoTexto (TST). Trata-se de uma teoria lexicográfica de base semântica que lança mão da Lexicografia Explicativa e Combinatória (LEC) para gerar produtos que ofereçam uma descrição linguística complexa e bastante completa do funcionamento das unidades lexicais no âmbito de um sistema linguístico, seja pensando a descrição de uma língua comum, seja suas diversas linguagens de especialidade, tanto em língua materna quanto em uma segunda língua. Dada a complexidade da TST, alguns estudiosos vêm se dedicando a apresentar uma linha mais didática de descrição linguística com 
foco na "popularização" de algumas ferramentas para a descrição do léxico de uma língua. Acompanhando essa corrente mais pedagógica das descrições linguísticas de lexias com base na TST, neste trabalho, foi analisado um exemplo de colocação lexical especializada (CLE) do domínio da Hemodinâmica com vistas a tornar a descrição do sentido linguístico mais acessível ao usuário-aprendiz.

Palavras-chave: Lexicografia Pedagógica. Lexicografia Explicativa e Combinatória. Colocações Lexicais Especializadas.

Abstract: This article is part of the studies about the use of pedagogical approaches to the lexicographic tools for describing specialized vocabulary when considering the lexical learning process. However, unlike proposing lexicographic products that aim to learn a second language, specifically, with a view to linguistic proficiency along the lines of foreign language teaching, in this article, we opted to try to use mechanisms already described in the specialized literature to present a pedagogical approach within the scope of the Meaning-Text Theory (TST). TST is a semantic-based lexicographic theory that uses Explanatory and Combinatory Lexicography (LEC) to generate products that offer a complex and quite complete linguistic description of the functioning of lexical units within a linguistic system, whether considering the description of a common language, be it its various specialty languages, both in the mother tongue and in a second language. Given the complexity of TST, the researches have been dedicated to presenting a more didactic line of linguistic description with a focus on the "popularization" of some TST tools for the description of the lexicon of a language. Accompanying this more pedagogical current of the linguistic descriptions of lexias based on TST, in this work, I analyze an example of specialized lexical collocation (CLE) in the domain of Hemodynamics in order to make the description of the linguistic sense more accessible to the user-learner.

Keyword: Pedagogical Lexicography. Explanatory and Combinatory Lexicography. Specialized Lexical Collocation.

\section{Considerações Iniciais}

Muitos são os estudos voltados para a aprendizagem de léxico de uma língua quando se fala em "Lexicografia Pedagógica". O intuito principal desses estudos é proporcionar ao aprendiz ferramentas linguísticas para a descrição do léxico de uma língua com o objetivo de suprir as necessidades desse aprendiz em seu processo de aprendizagem, relativamente ao que concerne às tarefas de produção e de compreensão de uma língua-alvo. No entanto, uma ampla parcela desses estudos é voltada para a aquisição do léxico de uma segunda língua. Por outro lado, a lexicografia pedagógica também propicia estudos que refletem a aplicabilidade do valor pedagógico de uma teoria lexicográfica, isso permite uma descrição linguística do léxico em língua materna e de suas diferentes linguagens de especialidade, ou melhor, dos vocabulários que caracterizam determinada área do conhecimento técnico-científico com vistas a uma aproximação descrição-usuário.

A escolha da Teoria Sentido-Texto (TST) como aporte teórico para refletir sobre práticas lexicográficas pedagógicas decorre do 
v. 11 (esp.)

180-195 ago. 2021

estudo presente na dissertação de mestrado de PIRES (2016), que teve como base essa mesma teoria. Aquele estudo levou à compreensão do grande potencial descritivo da TST. Mais especificamente, na pesquisa realizada para a dissertação, intitulada Colocações Lexicais Especializadas de base nominais no domínio da Hemodinâmica: um estudo exploratório na perspectiva da Teoria Sentido-Texto (PIRES, 2016), o objetivo foi identificar e analisar, semanticamente, as colocações lexicais especializadas (CLEs) do vocabulário da Hemodinâmica; realizando, assim, um exercício exploratório no qual se identificou as funções lexicais (FLs) que caracterizam as CLEs daquele domínio. O intuito foi observar a aplicabilidade das FLs propostas pela TST para a descrição do sentido das CLEs e, para aquele estudo, partiu-se da perspectiva de L'Homme $(1998,2001)$.

A TST é uma teoria lexicográfica de base semântica, iniciada em 1965, que tem por princípio a descrição lexical de línguas naturais, que se dá por meio de uma máquina lógica virtual que simula a atividade linguística do falante nativo de uma língua natural (Locutor), chamada de Modelo Sentido-Texto, tendo a tradução automática como finalidade primeira. Ademais, a valorosa aplicação da TST no Processamento da Linguagem Natural (PLN) (MILIĆEVIĆ, 2006, p.1-3) proporcionou o desenvolvimento de produtos lexicográficos que visam à exaustiva descrição linguística, os chamados Dicionários Explicativos e Combinatórios (DECs). Devido à complexidade descritiva da teoria e com o aumento do número de ferramentas e produtos lexicográficos desenvolvidos com base na TST com a finalidade da "popularização"1 dessa descrição, mais recentemente, houve a necessidade da TST apresentar uma perspectiva de cunho pedagógico para esses produtos e ferramentas lexicográficas, ofertando ao usuário desses produtos uma descrição linguística mais acessível, se comparada à exaustiva análise linguística presente nos DECs. Esse viés didático está presente, por exemplo, nos estudos e projetos de Mel'čuk \& Polguère (cf. POLGUÈRE; MEL'ČUK, 2007, POLGUÈRE, 2007), cujo trabalho tem foco na análise do léxico da língua comum francesa, e nas pesquisas e plataformas virtuais de L'Homme, desenvolvidas a partir de $2013^{2}$, focadas nos vocabulários especializados.

\footnotetext{
${ }^{1}$ O vocábulo "popularização", aqui, está sendo usado com o sentido 'permitir que a descrição linguística proposta pela teoria (TST) atinja tanto usuários leigos quanto especialistas'.

2 Plataformas do Observatoire de Linguistique Sens-Text (OLST), desenvolvidas por L'Homme et al., a partir de 2013. Disponível em: http://olst.ling.umontreal.ca/.
} 
Além disso, neste artigo a escolha pelo referencial teórico citado acima, nomeadamente perspectiva pedagógica da TST tem por finalidade, então, unir duas situações bem específicas: (i) a primeira relaciona-se com o fato de que serão utilizados, na análise proposta, alguns dos resultados obtidos no exercício exploratório desenvolvido para a dissertação de mestrado, que tinha por objetivo mostrar as combinatórias lexicais e as FLs que caracterizam CLEs do vocabulário da Hemodinâmica; e, (ii) em segundo lugar, será aplicada em um exemplo de CLE (ESTENOSE AÓRTICA) ${ }^{3}$ a lexicografia pedagógica da TST como entendida hoje, com vistas a facilitar o acesso de usuários aprendizes à descrição lexicográfica feita por essa teoria; sendo a proposta deste artigo, então, entre tantos já feitos por outros autores para finalidades diversas, um exercício da aplicação da lexicografia pedagógica na perspectiva da TST.

Em essência, a intenção aqui é pensar o uso de abordagens pedagógicas no que se refere às ferramentas lexicográficas de descrição de léxico, quando considerado o processo de aprendizagem lexical em língua materna. Isso porque, no doutorado, estamos desenvolvendo uma ferramenta lexicográfica que visa à aprendizagem de um vocabulário de uma linguagem especializada. Tal objetivo, então, faz deste estudo uma manobra exploratória de testagem da vertente pedagógica da TST, teoria lexicográfica escolhida para a implementação da descrição das lexias que serão disponibilizadas na ferramenta.

Nestes termos, a vertente pedagógica da TST tem por intuito "simplificar" a descrição lexicográfica presente na Lexicografia Explicativa e Combinatória (LEC) ${ }^{4}$, cujo principal produto são os Dicionários Explicativos e Combinatórios (DECs). Estes apresentam uma descrição exaustiva e complexa das lexias de uma língua, o que torna, muitas vezes, difícil sua compreensão descritiva, dando vazão de compreensão apenas ao linguista iniciado em TST. Por conta disso, a vertente pedagógica tem por finalidade "popularizar" a descrição lexicográfica a fim de aproximar os usuários/aprendizes de língua à descrição linguística proposta pela LEC.

Em síntese, diferentemente do que pretendemos com a tese de doutorado, que está em processo de desenvolvimento, a atividade

${ }_{3}$ Pelo formalismo da TST, quando registramos uma unidade lexical para descrição lexicográfica, a unidade analisada vem em caixa alta, em fonte menor que o texto.

4 A Lexicografia Explicativa e Combinatória nada mais é que o produto da modelização linguística dada pelo Modelo Sentido-Texto (Lexicologia Explicativa e Combinatória), ou seja, são os materiais produzidos a partir da descrição do léxico de uma língua via TST. 
v. 11 (esp.)

180-195 ago. 2021

proposta neste artigo usa como dados de análise alguns resultados obtidos no exercício exploratório realizado por PIRES (2016), que, como já anunciado, tinha por intuito descrever as colocações lexicais especializadas (CLE) do domínio da Hemodinâmica e mostrar as Funções Lexicais (FLs) que caracterizavam o domínio. Agora, no presente artigo, a intenção é realizar uma descrição lexicográfica, via perspectiva pedagógica da TST, em uma das CLEs já estudadas, no caso, ESTENOSE AÓRTICA.

Isso posto, e a fim de delinear o percurso que pretendemos seguir para desenvolver o presente exercício de análise da CLE ESTENOSE AÓRTICA, antes de adentrar na análise propriamente dita, julgamos necessário descrever brevemente o que caracteriza uma colocação lexical especializada (CLE), lexia a qual aplicaremos o exercício de testagem proposto neste artigo.

\section{Colocações Lexicais Especializadas (CLEs) segundo L'Homme (1998, 2001)}

De acordo com L'Homme (1998, 2001), uma CLE é uma combinação de lexemas cuja base ou núcleo é um termo e os elementos que a caracterizam são seus colocados / coocorrentes / argumentos (cf. TRATAMENTO MÉDICO, TRATAMENTO DE DADOS, em que TRATAMENTO é a base e MÉDICO e DADOS são colocados). CLEs são consideradas, segundo L'Homme (1998), estruturas sintagmáticas que possuem caráter composicional ou semicomposicional, ou seja, o valor do todo é a soma total ou parcial dos valores de suas partes. O significado da base é o pivô ou eixo do sentido da colocação, isso quer dizer que o significado "primitivo" da colocação está atrelado a sua base. Quanto a CLEs da Hemodinâmica estudadas por Pires (2016, p.89), a autora identificou que as colocações de área de especialidade apresentaram características específicas, sendo elas:

(i) CLEs têm extensão de 2 a 5 elementos (cf. CATETERDILATADOR (2), ANGIOPLASTIA PERCUTÂNEA CORONÁRIA (3), VALVOPLASTIA AÓRTICA POR CATETER-BALÃO (4) e VALVULOPLASTIA MITRAL PERCUTÂNEA POR CATETER-BALÃO (5));

(ii) CLEs possuem base nominal;

(iii)Os colocados são adjetivais (cf. CATETER DILATADOR), na maioria, e preposicionais (cf. ANGIOPLASTIA DE URGÊNCIA); (iv) Há presença de FLs adjetivais (maioria) e preposicionais; 
(v) Há presença de FL simples na descrição das CLEs (FLs standard); e

(vi) Há necessidade de acréscimo de informação à FL (houve acréscimo de informações para completar o sentido da definição para expressar a relação semântica existente entre os elementos da CLE), ou seja, para a descrição completa da relação semântica existente foi preciso lançar mão do uso de FL não-standard (PIRES, 2016, p.89).

Destacar o que são CLEs se faz necessário para deixar explícito o tipo de unidade lexical (lexia) que estamos analisando, uma vez que, quanto ao seu estatuto, CLEs são um subgrupo das colocações presentes na tipologia de frasemas proposta pela TST. Segundo Mel'čuk (2012, 2015), de acordo com a tipologia dos frasemas, essas lexias podem ser lexicais e semântico-lexicais. Os lexicais referem-se às estruturas que apresentam um sentido que é construído livremente pelo falante; mas seus lexemas constituintes são selecionados de forma restrita (cf. bater um bolão) e são não-composicionais (opacos); logo, por serem lexicais, configuram como unidades lexicais plenas, e a esse tipo fazem parte as locuções ou idiomatismos (cf. chutar o pau da barraca). Já os frasemas do tipo semântico-lexical são aqueles cujo sentido e lexemas são selecionados de maneira restrita (cf. por outro lado), são composicionais (transparentes) e a esse tipo pertencem as colocações (cf. educação básica), nominemas (cf. Cristo Redentor) e clichês (cf. quer casar comigo?). Por essa razão, estes últimos não são considerados unidades lexicais plenas, no sentido de que as colocações, por exemplo, são vistas, pela TST, como algo que faz parte da língua, uma forma de materialidade linguística; ou os clichês que são formas sociais com esvaziamento semântico. Assim, frasemas lexicais e semântico-lexicais se diferenciam pela natureza das restrições e pela composicionalidade. Isso acarreta que apenas locuções (idiomatismos) são entradas lexicais no DEC.

Isto posto, a fim de atestar esta proposta de aplicação da vertente pedagógica da TST, selecionamos uma CLE característica do domínio da Hemodinâmica com base nominal e complemento (colocado) adjetival, já que esse tipo de colocado apresentou maior frequência em CLEs da Hemodinâmica (cf. PIRES, 2016). Metodologicamente, sobre a seleção das CLEs da Hemodinâmica para o trabalho de 2016, cabe esclarecer que foram utilizados os Léxicos Realiter como fonte. Estes léxicos incluem glossários multilíngues para o tratamento de diferentes 
v. 11 (esp.)

180-195 ago. 2021

áreas de especialidade, donde selecionamos o Vocabulário Panlatino da Hemodinâmica ${ }^{5}$ para a identificação dos núcleos terminológicos da área e, a partir deles, localizar as CLEs em contextos provindos de artigos científicos da plataforma SciELO ${ }^{6}$. Por fim, as definições das CLEs foram retiradas do Compacto Dicionário Ilustrado de Saúde (SILVA; SILVA; VIANA, 2007).

Ademais, para a análise descritiva das CLEs, lançamos mão das FLs que, para Mel'čuk (1997), descrevem, em sentido matemático $\mathrm{f}(\mathrm{x})=\mathrm{y}$, as relações lexicais existentes entre os componentes de uma lexia. Essas relações podem ocorrer de duas formas: (i) pelo eixo paradigmático, que mantém uma forte ligação com o dado semântico; e (ii) pelo eixo sintagmático, que descreve a combinatória de unidades lexicais em sintagmas. Essas relações lexicais são encontradas em todas as línguas naturais modeladas pelo MST, sendo representadas na teoria pelas FLs. Assim, as FLs formalizam relações semânticas que representam significados gerais considerados básicos, muito perto do valor semântico primitivo expresso pela relação.

Nesse sentido, as FLs são uma ferramenta formal que visa à descrição compacta e sistemática de todos os tipos de relações lexicais existentes nas lexias de qualquer língua; sendo que uma FL aplicável a várias unidades lexicais é chamada de padrão ou standard; e a FL aplicável a apenas uma unidade lexical, ou a poucas unidades lexicais fechadas semanticamente é chamada de degenerada ou não-standard. Destacamos que, para encerrar essa seção, pela TST, as relações semânticas existentes entre os elementos de colocações da língua comum são representadas pela função simples do tipo $\mathrm{f}(\mathrm{x})=\mathrm{y}$. Quanto às CLEs, em razão de sua diferente natureza, optamos por representar as FLs por meio de variáveis que influenciam a função simples, como comentaremos mais adiante.

Contextualizadas as unidades lexicais que servirão de objeto deste exercício, passemos à análise propriamente dita.

\section{Exercício Exploratório: análise de ESTENOSE AÓRTICA}

Para realizar o exercício proposto por este artigo, selecionamos uma das CLEs mais representativas do domínio da Hemodinâmica. Para tanto, retomamos aos dados da dissertação para identificar critérios

\footnotetext{
${ }^{5}$ Disponível em http://www.realiter.net/lessici-realiter?lang=pt.

${ }^{6}$ Scientific Electronic Library Online Brazil, disponível em www.scielo.br.
} 
de seleção daquilo que seria considerado como uma CLE típica da área, assim, para a escolha da CLE a ser analisada, levamos em conta as seguintes informações: (i) 59,4\% das CLEs da Hemodinâmica apresentam dois elementos, são binárias, com a estrutura base + argumento; e (ii) a totalidade das CLEs binárias apresentam a estrutura Base Substantivo + Adjetivo (PIRES, 2016, p.70). A partir disso, optamos pela CLE ESTENOSE AÓRTICA que se encaixa nos critérios por ser uma CLE de base nominal, binária, cujo complemento ou colocado é adjetival.

O passo seguinte foi observar as FLs aplicáveis à lexia ESTENOSE AÓRTICA. Dessa maneira, identificamos, primeiramente, as FLs standard, paradigmática e sintagmática presentes na relação semântica existente entre os elementos constituintes da CLE. As FL standard são aquelas que expressam as relações semânticas mais comuns, são aplicadas em muitas lexias e são consideradas universais. A tabela 1, a seguir, contém esses dados quanto as FLs standard paradigmáticas e sintagmáticas identificadas na CLE ESTENOSE AÓRTICA (PIRES, 2016, p. 74 e 76):

\begin{tabular}{|c|c|c|}
\hline \multicolumn{3}{|c|}{ ESTENOSE AÓRTICA } \\
\hline EIXO & RELAÇÃO & $\begin{array}{l}\text { APLICAÇÃO } \\
\text { DA FL }\end{array}$ \\
\hline Sintagmático & $\begin{array}{l}\text { Adjetivo } \\
\text { intensificador }\end{array}$ & $\begin{array}{l}\text { Magn } \\
\text { (estenose } \\
\text { aórtica) = } \\
\text { grave, severa, } \\
\text { etc. }\end{array}$ \\
\hline Paradigmático & $\begin{array}{l}\text { Verbalização } \\
\text { (derivação estrutural/ } \\
\text { sintática) }\end{array}$ & $\begin{array}{l}\mathrm{V}_{o} \text { (estenose } \\
\text { aórtica) }= \\
\text { estenosar } \\
{[\sim \text { produzir }} \\
\text { estenose em] }\end{array}$ \\
\hline
\end{tabular}

As FLs sintagmáticas são aquelas que descrevem as combinações de elementos no nível do sintagma e expressam relações nominais, adjetivais/adverbiais, preposicionais e verbais. No exemplo, a CLE ESTENOSE AÓRTICA, no eixo sintagmático, apresenta uma relação adjetiva com valor intensificador que é expresso pela FL Magn. Isso quer dizer que o valor intensificador não é expresso de forma gratuita, pois depende da unidade lexical a partir da qual o sentido é expresso. Sabemos que esse valor é dado em sentido matemático por meio de uma função f que está associada a qualquer x para o qual podem ser expressos todos os valores possíveis de $\mathrm{y}$, assim, $\mathrm{f}(\mathrm{x})=\mathrm{y}$. Buscamos, para a unidade lexical $\mathrm{x}, \mathrm{o}(\mathrm{s})$ coocorrente(s) / colocado(s) que são os 
v. 11 (esp.)

180-195 ago. 2021

valores de y e argumentos de $\mathrm{x}$. Para melhor compreendermos essa relação, vejamos um exemplo de aplicação das FLs:

Exemplo: o valor 'intensidade' pode ser extraído da relação $\mathrm{f}(\mathrm{x})=\mathrm{y} / /$ 'intensidade' (chover) $=$ forte, torrencialmente, $\sim$ para caramba, a 'intensidade', aqui, é expressa por uma função $f$ (no caso Magn), chover é o valor $\mathrm{x}$, e forte, torrencialmente, para caramba são os valores de $\mathrm{y}$.

Pela TST, o sentido 'intensidade' é representado pela função Magn e a relação existente entre os elementos da lexia é expressa por Magn (chover) $=$ forte, torrencialmente, $\sim$ para caramba. Isso também ocorre com a CLE ESTENOSE AÓRTICA, no eixo sintagmático, a relação é adjetiva com valor de intensificação e é dada pela expressão Magn (estenose aórtica $)$ grave, severa, etc., em que Magn é a FL, ESTENOSE AÓRTICA é o x (base) e grave, severa, etc. são os valores de y (colocados/ coocorrentes).

No eixo paradigmático, isto é, aquele que mantém forte ligação com o dado semântico, as FLs expressas representam relações semânticas basilares (sinonímia, antonímia, hiperonímia), derivativos sintáticos ou estruturais (nominalizações, adjetivações, verbalizações) e derivativos semânticos (substantivos actantes, adjetivos actantes, advérbios actantes). No exemplo, a CLE ESTENOSE AÓRTICA, no eixo paradigmático, apresenta uma derivação estrutural ou sintática com o valor de verbalização que é expresso pela $F L V_{0}$. Assim, em $f(x)=y, V_{0}$ é a função (verbalização), ESTENOSE AÓRTICA é x, e estenosar [ produzir estenose em] são os valores de $\mathrm{y}$, expressos pela relação $\mathrm{V}_{\mathrm{o}}$ (estenose aórtica $)=$ estenosar [ produzir estenose em].

Apesar da identificação de FLs standard na CLE ESTENOSEAÓRTICA, diferentemente do que ocorre com unidades lexicais da língua comum que frequentemente podem ser empregadas em contextos diversificados, assumindo diferentes sentidos, nas linguagens de especialidade, as unidades lexicais tendem a ser empregadas em contextos específicos apresentando, muitas vezes, valores singulares. CLEs são colocações típicas de áreas de especialidade, logo, as FLs que melhor representam as relações expressas por esse tipo de unidade lexical são as FLs nãostandard, ou seja, aquelas que representam relações semânticas mais raras, típicas de linguagens particularizadas e utilizadas em contexto específico (L'HOMME, 1998). Assim, a tabela 2 a seguir traz a análise 
da CLE ESTENOSE AÓRTICA por meio da FL não-standard expressa pelos elementos constituintes da colocação (PIRES, 2016, p.102):

\begin{tabular}{c|c} 
Tabela 2 - Funções Lexicais Não-Standard \\
\hline \multicolumn{1}{|c}{ ESTENOSE AÓRTICA } \\
\hline Definição & $\begin{array}{c}\text { Estreitamento da } \\
\text { aorta }\end{array}$ \\
\cline { 2 - 2 } Função Lexical & $\begin{array}{c}{[\mathbf{z}(\mathbf{X}+\mathbf{Y}+\mathbf{Z})+\mathbf{f}(\mathbf{x})} \\
\mathbf{y}]\end{array}$ \\
\cline { 2 - 2 } & $\begin{array}{c}\text { : } \text { (doença X } \\
\text { provocada por Y } \\
\text { causadora de Z) } \\
+ \text { Ver (aórtica) }= \\
\text { estenose }\end{array}$
\end{tabular}

Fonte: Adaptado de PIRES, 2016 (p.102)

Como vemos na tabela 2 acima, ao usar FLs não-standard para formalizar a relação semântica existente entre os elementos que compõem uma CLE, foi necessário o acréscimo de informações às FLs para complementar o sentido expresso pelas colocações do domínio da Hemodinâmica. Comumente, as FLs têm sua formalização por meio de função simples $\mathrm{f}(\mathrm{x})$ = y; no entanto, em CLEs, pelo fato de ser necessário o uso de FLs não-standard e o acréscimo de informações para completar o sentido expresso pela relação presente entre os elementos da colocação, a formalização não poderia ser dada apenas por uma função simples do tipo $\mathrm{f}(\mathrm{x})=\mathrm{y}$. Devemos considerar, para uma compreensão do valor semântico da relação presente na colocação, tanto o tipo de colocado/ coocorrente que caracteriza o núcleo terminológico da CLE, quanto a informação extra que especifica a relação entre os elementos formativos da colocação. Isso acarreta a atuação de dois fatores conjuntos para a identificação da relação semântica existente; ou seja, para a formalização, o tipo de vínculo entre o colocado e a base é dado pela função $f(x)=$ y e a informação que acrescenta significado à função é dada pela variável z7 .

Na tabela 2, são observadas as seguintes informações: (i) a definição, adaptada do Compacto Dicionário Ilustrado de Saúde (SILVA; SILVA; VIANA, 2007); e (ii) a função lexical, expressa pela formalização $[\mathrm{z}(\mathrm{X}+\mathrm{Y}+\mathrm{Z})+\mathrm{f}(\mathrm{x})=\mathrm{y}]$, que na CLE é dada pela variável ' $\mathrm{z}$ ' somada à $\mathrm{f}(\mathrm{x})=\mathrm{y}$. Assim, a variável $\mathrm{z}$ é representada pela informação 'doença

\footnotetext{
${ }_{7}^{7}$ Cabe destacar que, para a dissertação de mestrado, consideramos a informação acrescida à $\mathrm{f}(\mathrm{x})$ = y também como uma função, ou seja, para aquele estudo, ponderamos a atuação de uma função do tipo composta $(g+f)(x)=g(x)+f(x)=y$, em que a informação acrescentada para completar o sentido dado pela CLE é representada por g(x) (cf. PIRES, 2016, p.81). No entanto, hoje, ao estudar a teoria de forma mais aprofundada, percebemos que, segundo a teoria, é mais apropriado tratar essa informação adicionada como uma variável que atua sobre a $f(x)=y$.
} 
v. 11 (esp.)

180-195 ago. 2021

X provocada por Y causadora de Z', a f(x) é Ver (função adjetiva), x é estenose (base da colocação) e y é aórtica (colocado da colocação).

De posse das informações apresentadas até então, imprescindíveis para a construção de uma ferramenta lexicográfica com base na LEC, passemos, finalmente, para a proposta de descrição da CLE ESTENOSE AÓRTICA por meio da lexicografia pedagógica via TST. Cabe destacar que a descrição de colocações não configura entradas em dicionários, mas aparecem no corpo do verbete, no entanto, a proposta aqui não é propor um verbete de dicionário, mas aplicar elementos de "simplificação" da análise linguística via LEC na descrição de colocações de área de especialidade. Dito isso, com base nas propostas de Polguère (2007), Polguère \& Mel'čuk (2007) e L'Homme et al. (2013), propomos uma análise que apresente uma revisão da nomenclatura bem como da maneira como a informação é apresentada ao usuário-aprendiz, uma vez que, temos por intenção, no doutorado, desenvolver uma ferramenta lexicográfica para a aprendizagem terminológica de léxico especializado para aprendizes de um curso técnico integrado ao ensino médio na modalidade EJA.

O presente artigo, então, representa um movimento exploratório que tem por finalidade subsidiar o percurso que temos traçado para pensar a criação dessa ferramenta lexicográfica, sendo um exercício de extrema importância para contribuir com a tese que estamos desenvolvendo. Sabe-se que a vertente pedagógica da TST tem por intuito "simplificar" para "popularizar" a descrição lexicográfica com a finalidade de aproximar os usuários/aprendizes à descrição linguística proposta pela LEC. Isso permite ao usuário melhor compreensão da descrição lexicográfica ofertada por ser uma descrição linguística adequada, diferente do DEC que traz uma descrição completa e complexa da lexia.

Um artigo do LAF de Polguère \& Mel'čuk (2007), por exemplo, apresenta uma adequação que supre as necessidades do usuário com informações necessárias e suficientes para a compreensão da identificação gramatical da lexia, seus valores polissêmicos, bem como as acepções que descrevem e servem de abonação para cada um de seus significados. L'Homme et al. (2013), por sua vez, desenvolveu plataformas para determinadas áreas do conhecimento que permitem a "simplificação" da modelização descritiva, seja pela forma como as informações estão apresentadas, seja por uso de recursos, como o visual, para facilitar a compreensão do usuário a fim de facilitar a disseminação do conhecimento. 
Destacamos, neste ponto, que pensar a aplicação da LEC para desenvolver produtos lexicográficos que visem à aprendizagem de língua materna por meio da descrição de colocações não é novidade. Neste sentido, podemos citar o trabalho de Borba (2018), que propõe a descrição de colocações do espanhol por meio da verificação das FLs presentes em colocações com a base hambre (fome). Naquele exercício, Borba (2018) demostrou o quanto descrições lexicográficas sob o viés pedagógico da TST podem ser adequadas para aprendizagem de colocações, uma vez que tal descrição, segundo Borba (2018, p.170)

[...] tem muito a oferecer a práxis lexicográfica, pois a sua metodologia elenca fatos de combinatória léxica idiossincráticos do espanhol. Trata-se de um aspecto da língua em uso a cuja descrição o aprendiz precisa ter acesso para que possa valer-se de um auxílio na produção de textos que tenham o 'jeito' do espanhol. (BORBA, 2018, p.170).

A novidade deste artigo, em relação ao trabalho de Borba (2018), está no fato de que estamos observando colocações típicas de linguagem especializada, diferentes das colocações presentes no léxico da língua comum observadas por Borba (2018). Assim, para um produto lexicográfico, visando à descrição de CLEs, que se proponha à aprendizagem de vocabulário técnico-científico, é preciso que se observe tanto produtos lexicográficos que se prestem à aprendizagem de léxico da língua comum, quanto os produtos criados para a aprendizagem de léxico de linguagens de especialidade. Os primeiros, por tenderem a propor um viés lexicográfico mais pedagógico, pensados de forma a facilitar e simplificar a aprendizagem de léxico por parte do aprendiz; os segundos, por promoverem uma "popularização" do conhecimento técnico-científico, descrevendo a unidade lexical terminológica através de especificidades pensadas para aproximar o usuário leigo à terminologia específica. A partir disso, propomos para a CLE ESTENOSE AÓRTICA, do âmbito da Hemodinâmica, a seguinte configuração de sua descrição: 
v. 11 (esp.)

180-195 ago. 2021

Quadro 1 - Modelização de CLE pela LEC para fins didáticos

ESTENOSE AÓRTICA

Definição Simplificada: Estreitamento da aorta

Definição Completa: Doença provocada por envelhecimento ou, em pessoa com menos de 70 anos, por defeito congênito, que causa o estreitamento da válvula aórtica

Tratamento: cirurgia, por meio da troca da válvula deficiente por uma nova que pode ser de material artificial ou natural (tecido suíno ou bovino)

Outros valores associados à ESTENOSE AÓRTICA:

a) Verbo: estenosar, [ produzir estenose em] (ex. um procedimento para não estenosar a artéria // um procedimento para não produzir estenose na artéria)

b) Adjetivos intensificadores: estenose aórtica grave, estenose aórtica severa, etc.

Contextos:

A estenose aórtica é um estreitamento da abertura da válvula aórtica que bloqueia (obstrui) o fluxo de sangue do ventrículo esquerdo para a aorta. A causa mais comum em pessoas... (Disponível em: https:// www.msdmanuals.com/pt-br/casa/SearchResults?query=Estenose+a \%c3\%b3rtica\&icd9=424.1\%3b395.0)

Estenose aórtica severa, em paciente assintomático e disfunção sistólica do ventrículo esquerdo (Fração de ejeção <50\%) sem ser por outra causa a não ser a estenose. Disponível em: https://www. cardiosurgerypost.com/

A estenose valvar aórtica é cada vez mais prevalente concordante com o envelhecimento populacional. Por conseguinte, torna-se mais comum o atendimento de pacientes assintomáticos com estenose aórtica grave. Disponível em: http://www.scielo.br/scielo.php?script=sci_ arttext\&pid=S0066- 782X2010001400019 Fonte: Elaborado pela própria autora a partir da análise dos dados de Pires (2016).

O Quadro 1 acima apresenta a configuração de modelização de uma CLE pela LEC para fins didáticos a qual nos propomos neste exercício. Antes de mais nada, imaginemos que o usuário do material aqui proposto, isto é, que o produto lexicográfico seja pensado para 
alunos de um curso técnico integrado ao ensino médio no qual os alunos pretendem seguir a área técnica profissionalmente. No exercício, o objetivo é "simplificar" a descrição lexicográfica ofertada pela LEC de modo a aproximar o usuário/aprendiz, proporcionando melhor compreensão descritiva se comparada à descrição presente no DEC, por exemplo.

O intuito é favorecer a aprendizagem terminológica, uma vez que estamos apresentando a materialidade linguística de colocações presentes em áreas de especialidade. Alguns podem se perguntar o porquê de ESTENOSE AÓRTICA como colocação e não como unidade terminológica, ressalto que o núcleo aqui é estenose, e este se trata de um núcleo terminológico, o que caracteriza uma CLE, como já destacado neste artigo. Além disso, também é possível, dentro do âmbito da Hemodinâmica, identificar as CLEs ESTENOSE CORONARIANA, ESTENOSE VALVAR, ESTENOSE PULMONAR, ESTENOSE MITRAL, ESTENOSE URETRAL, por exemplo.

Isto posto, para a modelização da CLE via lexicografia pedagógica, lançamos mão de informações gramaticais básicas, nome feminino passivo de plural e a categoria morfológica dos elementos constituintes da CLE, base substantiva e colocado adjetivo. A informação seguinte é dada pela definição da CLE, donde propusemos uma versão mais simplificada e objetiva da definição 'estreitamento da aorta', elaborada a partir da leitura do Compacto Dicionário Ilustrado de Saúde (SILVA; SILVA; VIANA, 2007) e uma segunda definição, mais completa, pensada a partir da FL não-standard que a CLE apresenta, isto é, z (doença X provocada por Y causadora de Z) + Ver(aórtica) = estenose; assim, "Doença provocada por envelhecimento ou, em pessoa com menos de 70 anos, por defeito congênito, que causa o estreitamento da válvula aórtica". Pela representação, a definição completa é formada pela soma das informações dadas pela variável z e pela função Ver, assim, $\mathrm{X}$ é doença/estenose aórtica, Y é envelhecimento ou defeito congênito e $Z$ é estreitamento da aorta.

Outro dado que julgamos importante acrescentar foi a informação de tratamento, pois consideramos que o produto irá atingir usuários/aprendizes de uma terminologia específica da área que irão atuar, logo, necessitam não apenas de informações sobre a doença, mas também formas de tratamento para essa doença. Em seguida, sob o título de "Outros valores associados à ESTENOSE AÓRTICA" apresentamos as relações expressas pelas FLs standard paradigmáticas e 
v. 11 (esp.)

180-195 ago. 2021

sintagmáticas presentes entre os elementos da lexia, sob uma tentativa de "simplificação da nomenclatura" ao chamar de "valores associados" "verbal" e "adjetival" o que, na análise fina, são os valores da função paradigmática $\mathrm{V}_{0}$ (verbalização) e da função sintagmática Magn (adjetival intensificadora). Por fim, trazemos os contextos de uso que também servem de exemplos para ESTENOSE AÓRTICA, ESTENOSE AÓRTICA severa e ESTENOSE AÓRTICA grave. O exemplo com a verbalização estenosar e [ produzir estenose em], apresentam-se juntamente com a FL $V_{0}$.

\section{Considerações Finais}

Cabe ter claro que proporcionar ao usuário uma modelização da língua que parta do dado semântico (sentido), aquilo que o falante quer dizer, às possíveis formas de realizá-lo (texto), ou seja, as paráfrases possíveis de o falante dizer, permite ao usuário/aprendiz um entendimento do processo de síntese linguística do ponto de vista do Locutor em um ato comunicativo, uma vez que o objeto da linguística é a língua, o que acarreta uma reflexão sobre a finalidade desse ato comunicativo e consequente consciência linguística.

Ademais, como destaca Polguère (2007), o intuito nessa "popularização" não é oferecer ao usuário/aprendiz uma descrição completa e complexa das lexias de uma língua que seja uma "verdade" científica, mas munir o aprendiz de elementos que sejam indispensáveis para a LEC e para a compreensão daquilo que é essencial à teoria. Com isso, ao aprendiz, é ofertada uma descrição linguística suficiente para a compreensão do sentido da lexia que precisa/deseja conhecer.

\section{Referências}

BORBA, Laura Campos de. A Teoria Sentido-Texto e suas possibilidades de aplicação em dicionários de aprendizes do espanhol. In: Revista da Anpoll, v. 1, n. 45, p. 156-172, Florianópolis, Maio/Ago. 2018.

L'HOMME et. al. Observatoire de linguistique Sens-Text (OLST), plataformas desenvolvidas a partir de 2013. Disponível em: http://olst.ling.umontreal.ca/ Acesso em: 02 set. 2020

L'HOMME, Marie-Claude. Combinaisons Lexicales Specilisées: regroupement des mots clés par classes conceitualles. In: DAILLE, B.; WILLIAMS, G. (Org.). Journées d'étude de l'ATALA. La Collocation. Rapport de Recherche. Nantes: Institut de Recherche en Informatique de Nantes, p.19-22, 2001.

L'HOMME, Marie-Claude. Caractérisation des combinaisons lexicales spécialisées par rapport aux collocations de langue générale. In: EURALEX '98 PROCEEDINGS, p. 513-22, 1998. 
MEL'ČUK, Igor. Semantics: from meaning to text. Vol.3. Philadelphia: John Benjamins, 2015.

MEL'ČUK, Igor. Vers une linguistique Sens-Text. Leçon Inaugurale, Collège de France, Chaire Internationale, p. 1-43, 1997.

MILIĆEVIĆ, Jasmina. A short-guide to the Meaning-Text Linguistic Theory. In: Journal of Koralex, vol.8: 187-233, 2006.

PIRES, Caroline de Castro. Colocações Lexicais Especializadas de base nominais no domínio da hemodinâmica: um estudo exploratório na perspectiva da Teoria Sentido-Texto. Dissertação de mestrado. Instituto de Letras, UFRGS, 2016.

POLGUÈRE, Alain; MEL'ČUK, Igor. Lexique actif du français: L'apprentissage du vocabulaire fondé sur 20000 dérivations sémantiques et collocations du français. Paris: Duculot, 2007.

POLGUÉRE, Alain. Lessons from the Lexique actif du français. In: MTT 2007, Klagenfurt, May 21 - 24, 2007.

SILVA, Carlos Roberto Lyra da; SILVA, Roberto Carlos Lyra da; VIANA, Dirce Laplaca. Compacto Dicionário Ilustrado de Saúde. $2^{\mathrm{O}}$ edição, revisada e ampliada. Editora: Yendis, 2007. 